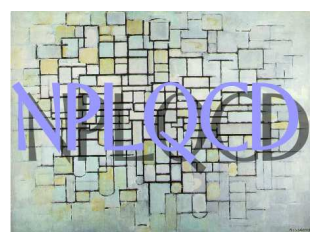

NT@UW-07-11

UNH-07-02

\title{
$n$-Boson Energies at Finite Volume and Three-Boson Interactions
}

\author{
Silas R. Beane, ${ }^{1}$ William Detmold, ${ }^{2}$ and Martin J. Savage ${ }^{2}$ \\ 1 Department of Physics, University of New Hampshire, Durham, NH 03824-3568, USA \\ ${ }^{2}$ Department of Physics, University of Washington, Box 351560, Seattle, WA 98195, USA
}

(Dated: November 5, 2018)

\begin{abstract}
We calculate the volume dependence of the ground-state energy of $n$ identical bosons with shortrange repulsive interactions in a periodic spatial volume of side $L$, up to and including terms of order $L^{-6}$. With this result, Lattice QCD calculations of the ground-state energies of three or more pions will allow for a systematic extraction of the three-pion interaction at this order in the volume expansion.
\end{abstract}

\section{INTRODUCTION}

A major goal of the field of strong-interaction physics is to determine the spectrum of hadrons and nuclei from Quantum Chromodynamics (QCD). Lattice QCD is the only known way to rigorously compute strong-interaction quantities, and as such, an increasing effort is being put into understanding the lattice QCD calculations that will be required to extract even the most basic properties of light nuclei. Ground-state hadron masses, including those of nuclei, can be extracted from the long-time exponential behavior of a two-point correlation function with the appropriate quantum numbers in Euclidean space. Excited states that are stable against strong decays, such as the low-lying energy levels of nuclei, can be extracted from the time-dependence of correlation functions over a large time interval with various methods, such as those described in Ref. [1, 2]. By contrast, excited states that are unstable against strong decays can be extracted by analyzing the volume dependence of the energies of the scattering states [3]. However, such direct calculations of the masses of nuclei beyond $A \sim 6$ appear computationally prohibitive using current lattice techniques. Consequently, the properties of nuclei, beyond the lightest few, will likely be systematically determined by matching lattice QCD onto nuclear effective field theories (NEFT's) ${ }^{1}$. Lattice QCD calculations of few nucleon systems will be used to constrain the low-energy constants (LECs) appearing in the NEFT, which will then be used to compute the properties of larger nuclei. To extend calculations to larger nuclei, further matchings (for example to the no-core shell model [7]) will be required. The most important LECs relate to the interactions in the two-body sector, characterized by the effective-range expansion of the two-particle phase shift. These contributions (at the physical pion mass) are well constrained by experiment, but can also be determined using lattice QCD. Recent work has used the volume dependence 8, 9, 10] of two nucleon 11] and hyperon-nucleon [12, 13] systems to extract the corresponding two-body low-energy phase shifts at specific momenta from fully-dynamical mixed-action lattice calculations (domain-wall valence quarks on fourth-rooted staggered configurations generated by the MILC collaboration). In principle, phase shifts for all momenta below inelastic thresholds can be determined from QCD given sufficient computational resources.

In order to perform NEFT computations in systems of more than two nucleons, $A>2$, it is necessary to include the multi-nucleon interactions, such as the three-body force. The leading, local three-body interaction is a momentumindependent contact operator, $\mathcal{O}^{3-\text { body }} \sim D(\mu)[\bar{N} N]^{3}$, where $\mu$ is the renormalization scale (which we will discuss subsequently), and $N$ is the nucleon annihilation operator. A determination of the coupling $D(\mu)$ from lattice QCD will require the study of three-nucleon systems. This is a complicated task, and aside from the numerical complexities of such a lattice calculation, theoretical developments are required in a number of relevant areas.

In this work, we compute the ground-state energy of $n$ identical bosons in a finite cubic volume with short-range repulsive two-body interactions, subject to periodic boundary conditions. This system was first investigated for hard

1 For reviews of NEFT see Refs. [4, [5, []]. 
spheres by Huang and Yang [14], and we extend their results to include order $L^{-6}$ contributions in the volume expansion, where the two-body effective range and three-body interactions first enter. For the case of three hardspheres, our result agrees with that of $\mathrm{Wu}$ [15] (modulo renormalization issues and the appearance of a three-body interaction). While our results are not directly relevant to the study of nuclei, they are necessary for extracting the three-boson interaction in the study of multi-boson systems (for instance multi-pion systems).

\section{THE GROUND STATE ENERGY OF $n$ BOSONS}

Motivated by interest in performing lattice QCD calculations, we calculate the ground-state energy of $n$ bosons of mass $M$ confined to a finite volume of size $L^{3}$ with periodic boundary conditions ${ }^{2}$ in non-relativistic quantum mechanics. In their classic 1957 paper, Huang and Yang [14] (see also Ref. [15, 16]) considered this problem formulated for hard spheres using the technique of pseudo-potentials. They calculated the dependence of the $n$-boson ground state on the volume up to $L^{-5}$ :

$$
E_{0}(n, L)=\frac{4 \pi a}{M L^{3}}\left\{\left(\begin{array}{c}
n \\
2
\end{array}\right)-\left(\frac{a}{\pi L}\right)\left(\begin{array}{l}
n \\
2
\end{array}\right) \mathcal{I}+\left(\frac{a}{\pi L}\right)^{2}\left\{\left(\begin{array}{l}
n \\
2
\end{array}\right) \mathcal{I}^{2}-\left[\left(\begin{array}{c}
n \\
2
\end{array}\right)^{2}-12\left(\begin{array}{l}
n \\
3
\end{array}\right)-6\left(\begin{array}{l}
n \\
4
\end{array}\right)\right] \mathcal{J}\right\}\right\}+\mathcal{O}\left(L^{-6}\right),(1)
$$

where the integer sums $\mathcal{I}$ and $\mathcal{J}$ are

$$
\mathcal{I}=\lim _{\Lambda_{j} \rightarrow \infty} \sum_{\mathbf{i} \neq \mathbf{0}}^{|\mathbf{i}| \leq \Lambda_{j}} \frac{1}{|\mathbf{i}|^{2}}-4 \pi \Lambda_{j}=-8.91363291781 \quad, \quad \mathcal{J}=\sum_{\mathbf{i} \neq \mathbf{0}} \frac{1}{|\mathbf{i}|^{4}}=16.532315959,
$$

where the sums extend over all three-vectors of integers, and $\left(\begin{array}{l}n \\ k\end{array}\right)=n ! /(n-k) ! / k !$. In eq. (1), the notation has been modified, and numerical values of $\mathcal{I}$ and $\mathcal{J}$ have been corrected, compared to the expressions presented in Refs. 14, 16]. The leading term in eq. (1) was derived by Bogoliubov [17]. In the special case of two particles, Lüscher [9, 10] has shown that the energy shift determined in non-relativistic quantum mechanics, given by eq. (1) with $n=2$, can also be derived from quantum field theory, and thus is a general result ${ }^{3}$.

At $\mathcal{O}\left(L^{-5}\right)$, the ground-state energy in eq. (11) is not sensitive to three-body interactions. On dimensional grounds, three-body contributions should first enter at $\mathcal{O}\left(L^{-6}\right)$ and in this work we extend the result given above to that order. This will allow for the extraction of three-body interactions from lattice QCD calculations. The ground-state energy of the $n$-boson system is calculated with an interaction of the form

$$
V\left(\mathbf{r}_{1}, \ldots, \mathbf{r}_{\mathbf{n}}\right)=\eta \sum_{i<j}^{n} \delta^{(3)}\left(\mathbf{r}_{i}-\mathbf{r}_{j}\right)+\eta \sum_{i<j<k} \delta^{(3)}\left(\mathbf{r}_{i}-\mathbf{r}_{k}\right) \delta^{(3)}\left(\mathbf{r}_{j}-\mathbf{r}_{k}\right)+\ldots
$$

where the ellipsis denote higher-body interactions that do not contribute at the order to which we work (in general, $m$-body interactions will enter at $\mathcal{O}\left(L^{3(1-m)}\right)$. For an $s$-wave scattering phase shift, $\delta(p)$, the two-body contribution to the pseudo-potential is given by $\eta=-\frac{4 \pi}{M} p^{-1} \tan \delta(p)=\frac{4 \pi}{M} a+\frac{\pi}{M} a^{2} r\left(\vec{p}^{2}+\overleftarrow{p}^{2}\right)+\ldots$, keeping only the contributions from the scattering length and effective range, $a$ and $r$, respectively. At $\mathcal{O}\left(L^{-6}\right)$ the coefficient of the three-body potential, $\eta_{3}$, is momentum independent. While up to this point the discussion has been phrased in terms of $m$-body pseudo-potentials, the modern language with which to describe these interactions and calculations is that of the pionless $\mathrm{EFT}, \operatorname{EFT}(\star)[22,23,24]$. In $\operatorname{EFT}(\hbar)$ the divergences that occur in loop diagrams can be renormalized orderby-order in the expansion, preserving the power counting. This is somewhat less obvious when using the language of pseudo-potentials.

At $\mathcal{O}\left(L^{-6}\right)$ (order $V^{4}$, corresponding to four insertions of the two-body potential given in eq. (3)) the energyshift has contributions with interactions amongst up to six particles. Using standard techniques of non-degenerate perturbation theory (or the perturbative expansion of EFT $(\hbar)$ ), the ground-state energy of $n$ bosons with repulsive

\footnotetext{
2 The effects of the finite time extent of lattice calculations are exponentially small in the temporal length and are ignored herein.

3 The two-body result has been extended to the situation where the center-of-mass is moving relative to the volume [18, 19, 20], and also to the case in which there are coupled channels [21].
} 
two-body interactions is

$$
\begin{aligned}
E_{0}(n, L)=\frac{4 \pi a}{M L^{3}}\left\{\left(\begin{array}{l}
n \\
2
\end{array}\right)-\left(\frac{a}{\pi L}\right)\left(\begin{array}{l}
n \\
2
\end{array}\right) \mathcal{I}+\left(\frac{a}{\pi L}\right)^{2}\left\{\left(\begin{array}{l}
n \\
2
\end{array}\right) \mathcal{I}^{2}-\left[\left(\begin{array}{l}
n \\
2
\end{array}\right)^{2}-12\left(\begin{array}{l}
n \\
3
\end{array}\right)-6\left(\begin{array}{l}
n \\
4
\end{array}\right)\right] \mathcal{J}\right\}\right. \\
+\left(\frac{a}{\pi L}\right)^{3}\left[-\left(\begin{array}{l}
n \\
2
\end{array}\right) \mathcal{I}^{3}+3\left(\begin{array}{l}
n \\
2
\end{array}\right)^{2} \mathcal{I} \mathcal{J}-\left(\begin{array}{l}
n \\
2
\end{array}\right)^{3} \mathcal{K}-24\left(\begin{array}{c}
n \\
3
\end{array}\right)\left(\mathcal{I} \mathcal{J}+2 \mathcal{Q}+\mathcal{R}-\mathcal{K}\left(\begin{array}{c}
n \\
2
\end{array}\right)\right)\right. \\
\left.\left.-6\left(\begin{array}{l}
n \\
4
\end{array}\right)\left(3 \mathcal{I} \mathcal{J}+51 \mathcal{K}-2\left(\begin{array}{l}
n \\
2
\end{array}\right) \mathcal{K}\right)-300\left(\begin{array}{c}
n \\
5
\end{array}\right) \mathcal{K}-90\left(\begin{array}{l}
n \\
6
\end{array}\right) \mathcal{K}\right]\right\} \\
+\left(\begin{array}{l}
n \\
3
\end{array}\right) \frac{64 \pi a^{4}}{M L^{6}}(3 \sqrt{3}-4 \pi) \log (\mu L)+\left(\begin{array}{c}
n \\
2
\end{array}\right) \frac{8 \pi^{2} a^{3}}{M L^{6}} r+\left(\begin{array}{c}
n \\
3
\end{array}\right) \frac{\eta_{3}(\mu)}{L^{6}}+\mathcal{O}\left(L^{-7}\right)
\end{aligned}
$$

which can also be written as

$$
\begin{aligned}
E_{0}(n, L)=\frac{4 \pi a}{M L^{3}}\left(\begin{array}{l}
n \\
2
\end{array}\right)\{ & 1-\left(\frac{a}{\pi L}\right) \mathcal{I}+\left(\frac{a}{\pi L}\right)^{2}\left[\mathcal{I}^{2}+(2 n-5) \mathcal{J}\right] \\
& \left.+\left(\frac{a}{\pi L}\right)^{3}\left[-\mathcal{I}^{3}-(2 n-7) \mathcal{I} \mathcal{J}-\left(5 n^{2}-41 n+63\right) \mathcal{K}-8(n-2)(2 \mathcal{Q}+\mathcal{R})\right]\right\} \\
& +\left(\begin{array}{c}
n \\
3
\end{array}\right) \frac{64 \pi a^{4}}{M L^{6}}(3 \sqrt{3}-4 \pi) \log (\mu L)+\left(\begin{array}{c}
n \\
2
\end{array}\right) \frac{8 \pi^{2} a^{3}}{M L^{6}} r+\left(\begin{array}{c}
n \\
3
\end{array}\right) \frac{\eta_{3}(\mu)}{L^{6}}+\mathcal{O}\left(L^{-7}\right)
\end{aligned}
$$

In the case of $n=3$, the logarithmic behavior with volume was first derived by Wu [15], and the ground-state energy has recently been computed to $\mathcal{O}\left(L^{-7}\right)$ by S. Tan [25]. The additional integer sums that contribute at $\mathcal{O}\left(L^{-6}\right)$ are

$$
\begin{aligned}
\mathcal{K} & =\sum_{\mathbf{i} \neq \mathbf{0}} \frac{1}{|\mathbf{i}|^{6}}=8.401923974433 \\
\widehat{\mathcal{Q}} & =\sum_{\mathbf{i} \neq \mathbf{0}} \sum_{\mathbf{j} \neq \mathbf{0}} \frac{1}{|\mathbf{i}|^{2}|\mathbf{j}|^{2}\left(|\mathbf{i}|^{2}+|\mathbf{j}|^{2}+|\mathbf{i}+\mathbf{j}|^{2}\right)}, \\
\widehat{\mathcal{R}} & =\sum_{\mathbf{j} \neq \mathbf{0}} \frac{1}{|\mathbf{j}|^{4}}\left[\sum_{\mathbf{i}} \frac{1}{|\mathbf{i}|^{2}+|\mathbf{j}|^{2}+|\mathbf{i}+\mathbf{j}|^{2}}-\frac{1}{2} \int d^{d} \mathbf{i} \frac{1}{|\mathbf{i}|^{2}}\right] .
\end{aligned}
$$

The sums $\widehat{\mathcal{Q}}$ and $\widehat{\mathcal{R}}$ arise from intrinsic two-loop contributions to the energy and are naively divergent $(\widehat{\mathcal{R}}$ contains a nested divergence, requiring the subtraction to preserve the one-loop scattering amplitude). As is the case for $\mathcal{I}$, given in eq. (2), we define (regulate) these sums using dimensional regularization. It is straightforward to show that the dimensionally-regulated sums that appear in eq. (7) and eq. (8) are

$$
\begin{aligned}
& \widehat{\mathcal{Q}} \rightarrow \mathcal{Q}+\frac{4}{3} \pi^{4} \log (\mu L)-\frac{2 \pi^{4}}{3(d-3)}, \\
& \widehat{\mathcal{R}} \rightarrow \mathcal{R}-2 \sqrt{3} \pi^{3} \log (\mu L)+\frac{\sqrt{3} \pi^{3}}{d-3} .
\end{aligned}
$$

Numerically, we find that $\mathcal{Q}=-100.75569$ and $\mathcal{R}=19.186903$. In eq. (44) and in what follows from it, the MS-scheme is used to define the three-body coefficient, $\eta_{3}(\mu)$ in which only the pole, $\sim \frac{1}{d-3}$, is subtracted from the divergent two-loop diagrams (explicitly absorbed by $\eta_{3}(\mu)$ ). This renormalization, and the associated logarithmic dependence on $\mu L$ in eq. (4), make clear that the three-body interaction, $\eta_{3}(\mu)$, must enter at this order [26]; the scale dependence of $\eta_{3}(\mu)$ exactly cancels that of the logarithms in eq. (4) and in subsequent expressions. The logarithms of $\mu L$ that appear in eq. (4) result from the relation between the momentum-space integrals in $d$-dimensions and the integer sums. It is worth stressing that the quantities $\mathcal{Q}$ and $\mathcal{R}$ are subtraction-scheme dependent.

In the first form of our main result, eq. (4), the combinatoric factors make clear the number of particles involved in the various contributions; terms proportional to $\left(\begin{array}{l}n \\ j\end{array}\right)$ have $j$ interacting particles and $n-j$ spectators. The second, simpler form of our result, eq. (5), follows from evaluating the combinatoric factors. As can be seen from these expressions, at this order the two-body effective range also contributes. 
For two, three and four particles the ground-state energies are

$$
\begin{gathered}
E_{0}(2, L)=\frac{4 \pi a}{M L^{3}}\left\{1-\left(\frac{a}{\pi L}\right) \mathcal{I}+\left(\frac{a}{\pi L}\right)^{2}\left[\mathcal{I}^{2}-\mathcal{J}\right]+\left(\frac{a}{\pi L}\right)^{3}\left[-\mathcal{I}^{3}+3 \mathcal{I} \mathcal{J}-\mathcal{K}\right]\right\}+\frac{8 \pi^{2} a^{3}}{M L^{6}} r+\mathcal{O}\left(L^{-7}\right) \\
E_{0}(3, L)=\frac{12 \pi a}{M L^{3}}\left\{1-\left(\frac{a}{\pi L}\right) \mathcal{I}+\left(\frac{a}{\pi L}\right)^{2}\left[\mathcal{I}^{2}+\mathcal{J}\right]+\left(\frac{a}{\pi L}\right)^{3}\left[-\mathcal{I}^{3}+\mathcal{I} \mathcal{J}+15 \mathcal{K}-8(2 \mathcal{Q}+\mathcal{R})\right]\right\} \\
\quad+\frac{64 \pi a^{4}}{M L^{6}}(3 \sqrt{3}-4 \pi) \log (\mu L)+\frac{24 \pi^{2} a^{3}}{M L^{6}} r+\frac{1}{L^{6}} \eta_{3}(\mu)+\mathcal{O}\left(L^{-7}\right), \\
E_{0}(4, L)=\frac{24 \pi a}{M L^{3}}\left\{\begin{array}{c}
\left.1-\left(\frac{a}{\pi L}\right) \mathcal{I}+\left(\frac{a}{\pi L}\right)^{2}\left[\mathcal{I}^{2}+3 \mathcal{J}\right]+\left(\frac{a}{\pi L}\right)^{3}\left[-\mathcal{I}^{3}-\mathcal{I} \mathcal{J}+21 \mathcal{K}-16(2 \mathcal{Q}+\mathcal{R})\right]\right\} \\
+\frac{256 \pi a^{4}}{M L^{6}}(3 \sqrt{3}-4 \pi) \log (\mu L)+\frac{48 \pi^{2} a^{3}}{M L^{6}} r+\frac{4}{L^{6}} \eta_{3}(\mu)+\mathcal{O}\left(L^{-7}\right)
\end{array}\right.
\end{gathered}
$$

From eq.(4), it is possible to construct combinations of these energies that are directly sensitive to the three-body contributions at $L^{-6}$. That is, for $n>2$,

$$
\begin{aligned}
& E_{0}(n, L)-\left(\begin{array}{l}
n \\
2
\end{array}\right) E_{0}(2, L) \\
&-6\left(\begin{array}{l}
n \\
3
\end{array}\right) {\left[\frac{M^{2} L^{4}}{16 \pi^{4}} \mathcal{J}\left[E_{0}(2, L)\right]^{3}+\frac{M^{3} L^{6}}{32 \pi^{6}}\left(\mathcal{I} \mathcal{J}-\frac{1}{4} \mathcal{K}(5 n-31)-2(2 \mathcal{Q}+\mathcal{R})\right)\left[E_{0}(2, L)\right]^{4}\right] } \\
&=\left(\begin{array}{l}
n \\
3
\end{array}\right) \frac{1}{L^{6}}\left[\frac{64 \pi a^{4}}{M}(3 \sqrt{3}-4 \pi) \log (\mu L)+\eta_{3}(\mu)\right]+\mathcal{O}\left(L^{-7}\right)
\end{aligned}
$$

This can also be accomplished by forming judicious combinations of the energies of three different systems, such as $E_{0}(4, L), E_{0}(3, L)$ and $E_{0}(2, L)$. Further, combinations of energies can be formed in which the three-body interaction is absent, such as

$$
E_{0}(2, L)-\frac{2}{3} E_{0}(3, L)+\frac{1}{6} E_{0}(4, L)+40 \frac{M^{3} L^{6}}{256 \pi^{6}} \mathcal{K}\left[E_{0}(2, L)\right]^{4}=\mathcal{O}\left(L^{-7}\right)
$$

Relations such as that of eq. (15) will provide a useful check of both the statistical and systematic uncertainties associated with lattice QCD calculations.

The calculation of ground-state energies described here has been derived in a non-relativistic framework, however the results remain valid relativistically. In the two-body case, this has been shown by Lüscher [9]. In the higher-body case, the non-relativistic calculation will not correctly recover a field-theoretic calculation, due to relativistic effects in multiple, two-body interactions involving three or more particles. At $\mathcal{O}\left(L^{-4}\right)$, only two-particle interactions contribute to the $n$-body ground-state energy and the results of Ref. [9] follow without modification. Since the interaction of three particles due to the two-body interaction first enters at $L^{-5}$, and relativistic effects in such interactions are suppressed by $(M L)^{-2}$, the first relativistic effects will occur at $\mathcal{O}\left(L^{-7}\right)$. Generally, relativistic effects can be included perturbatively into the volume expansion by using $\operatorname{EFT}(\hbar)$ 22, 23, 24].

Beyond $\mathcal{O}\left(L^{-6}\right)$ a number of other effects are also important:

- Higher partial waves will contribute to the ground-state energy at finite volume. The cubic periodic boundary conditions imposed on the system are such that the spatial symmetry group is $H(3)$ with the ground-state wavefunction transforming in the $A_{1}^{+}$representation (see Ref. 27]). Contributions to the ground-state energy from interactions other than $s$-wave (as classified in the continuum) first enter at order $\mathcal{O}\left(L^{-7}\right)$ via three twobody interactions, two of which are $s$-wave and one of which is $p$-wave. The leading two-body contribution from higher partial-waves results from a single insertion of an $l=4$ operator, and enters at $\mathcal{O}\left(L^{-11}\right)$.

- It is straightforward to show that the leading, momentum-independent $m$-body operators contribute to the ground-state energy at $L^{3(1-m)}$, and therefore, the four-body local operator will first enter at order $L^{-9}$. It is obvious that the momentum-dependent $m$-body operators first enter two orders higher than the momentumindependent operators. For instance, contributions from the two-body shape parameters (higher-order terms in the expansion of $p \cot \delta(p))$ first occur at $\mathcal{O}\left(L^{-8}\right)$. 
- In the calculations that we have performed, the symmetrizations that are required to satisfy Bose statistics are trivial. At higher orders in the expansion, where multiple insertions of the two-body effective-range interaction occur, symmetrization of the intermediate states will have non-trivial consequences and calculations will become increasingly complicated.

\section{DISCUSSION AND CONCLUSION}

In this work we have computed the volume dependence of the ground-state energy of $n$ bosons in a cubic volume with periodic boundary conditions. Knowledge of this dependence is necessary in order to establish a connection between lattice QCD calculations of $n$-boson systems in Euclidean space and the multi-body interactions contributing to the properties of many-body systems in Minkowski space. We conclude by highlighting a number of issues and possible extensions of this work:

- Our results are easily generalized to asymmetric volumes. In the asymmetric case, the integer sums that appear in the expressions for the ground-state energy will become dependent on the asymmetry parameters [28, 29] (for some asymmetries, one or more of these sums can vanish [29]). Further, the reduced symmetry of the system forces derivative interactions and higher partial-wave interactions to enter at lower orders in the volume expansion [30].

- Calculation of excited-state energies are straightforward and tedious. Many contributions (diagrams in the perturbative expansion) that vanish in the calculation of the ground-state energy will no longer vanish, and the combinatoric factors become somewhat more complicated because of the symmetrization requirements.

- Our calculation of the ground-state energy of the $n$-boson system is an expansion whose terms depend parametrically on $n$ and $L$ as $n^{\alpha} / L^{\beta}$, where $\alpha$ and $\beta$ are positive integers. Much of the discussion in the literature regarding collections of bosons concerns Bose-condensed systems, with the results that are summarized in Ref. [31. It is clear that in the large- $n$ limit, relevant to the Bose-condensed systems, this expansion fails, and as such, no direct connection between our calculation and Bose-condensed systems can be made.

- We have restricted ourselves to the case of repulsive interactions among the bosons. The reason for this is that for an attractive two-body interaction of sufficient strength, the ground-state will not be perturbatively close to $n$ non-interacting bosons but will, in fact, be a system of two-body (or higher body) bound states, e.g. one can imagine that for the three-body system the ground-state is a two-body bound state interacting with the third boson. The finite-volume behavior of the two-particle system with shallow (compared to the inverse range of the underlying interaction) bound states was discussed in Ref. [32], and subsequently numerically explored in Ref. [33]. In such systems, the leading finite volume corrections have both power-law and exponential volume dependences, and are somewhat more complicated to analyze.

- The expressions we have presented in this work form the basis for investigating $\pi^{n}$ correlation functions with isospin $I=I_{z}=n$ in lattice QCD. By forming well-defined ratios of correlation functions, as given in eq. (14), the three-body interaction can be calculated. The natural choice of renormalization scale is the ultra-violet cutoff of the low-energy theory, which is approximately the range of the interaction, $\mu \sim r^{-1}$. This gives rise to a large $\log$ arithm, $\log (r L) / L^{6}$, which should formally dominate the $L^{-6}$ contribution to the ground-state energy.

- As the lattice-QCD study of nuclei is the underlying motivation for this work, it is worth considering difficulties that will be encountered in generalizing the result described here to fermionic systems. A significant difference between the three-neutron system and the three-boson system is that the Pauli-exclusion principle forces the unperturbed ground state of three neutrons to have non-zero momentum. One of the neutrons will have momentum $|\mathbf{p}|=2 \pi / L$, which provides power-law volume dependence to the ground state even in the absence of interactions. As a result the momentum-dependent interactions will enter at lower orders than in the boson system. The lowest-energy state with zero total momentum consists of one neutron at rest, and the other two moving back-to-back, each with $|\mathbf{p}|=2 \pi / L$.

\section{Acknowledgments}

We thank A. Bulgac, M. Forbes, S. Tan and A. Torok for useful discussions. The work of SRB was supported in part by National Science Foundation CAREER grant No. PHY-0645570 and that of WD and MJS by Department of 
Energy grant DE-FG03/974014.

[1] C. Michael, Nucl. Phys. B 259, 58 (1985).

[2] M. Lüscher and U. Wolff, Nucl. Phys. B 339, 222 (1990).

[3] M. Lüscher, Nucl. Phys. B 364, 237 (1991).

[4] S. R. Beane, P. F. Bedaque, W. C. Haxton, D. R. Phillips and M. J. Savage, arXiv:nucl-th/0008064.

[5] P. F. Bedaque and U. van Kolck, Ann. Rev. Nucl. Part. Sci. 52, 339 (2002) arXiv:nucl-th/0203055.

[6] E. Epelbaum, Prog. Part. Nucl. Phys. 57, 654 (2006) arXiv:nucl-th/0509032.

[7] C. Forssen, P. Navratil, W. E. Ormand and E. Caurier, Phys. Rev. C 71, 044312 (2005) arXiv:nucl-th/0412049.

[8] H. W. Hamber, E. Marinari, G. Parisi and C. Rebbi, Nucl. Phys. B 225, 475 (1983).

[9] M. Lüscher, Commun. Math. Phys. 105, 153 (1986).

[10] M. Lüscher, Nucl. Phys. B 354, 531 (1991).

[11] S. R. Beane, P. F. Bedaque, K. Orginos and M. J. Savage, Phys. Rev. Lett. 97, 012001 (2006) arXiv:hep-lat/0602010.

[12] S. R. Beane, P. F. Bedaque, A. Parreño and M. J. Savage, Nucl. Phys. A 747, 55 (2005) arXiv:nucl-th/0311027.

[13] S. R. Beane, P. F. Bedaque, T. C. Luu, K. Orginos, E. Pallante, A. Parreño and M. J. Savage [NPLQCD Collaboration], arXiv:hep-lat/0612026.

[14] K. Huang and C. N. Yang, Phys. Rev. 105, 767 (1957).

[15] T. T. Wu, Phys. Rev. 155, 1390 (1959).

[16] T. D. Lee, K. Huang, and C. N. Yang, Phys. Rev. 106, 1135 (1957)

[17] N. N. Bogoliubov, J. Phys. (Moscow) 11, 23 (1947).

[18] K. Rummukainen and S. A. Gottlieb, Nucl. Phys. B 450, 397 (1995) arXiv:hep-lat/9503028.

[19] C. h. Kim, C. T. Sachrajda and S. R. Sharpe, Nucl. Phys. B 727, 218 (2005) arXiv:hep-lat/0507006.

[20] N. H. Christ, C. Kim and T. Yamazaki, Phys. Rev. D 72, 114506 (2005) arXiv:hep-lat/0507009.

[21] S. He, X. Feng and C. Liu, JHEP 0507, 011 (2005) arXiv:hep-lat/0504019.

[22] D. B. Kaplan, M. J. Savage and M. B. Wise, Phys. Lett. B 424, 390 (1998) arXiv:nucl-th/9801034.

[23] U. van Kolck, Nucl. Phys. A 645, 273 (1999) arXiv:nucl-th/9808007.

[24] J. W. Chen, G. Rupak and M. J. Savage, Nucl. Phys. A 653, 386 (1999) arXiv:nucl-th/9902056.

[25] S. Tan, to appear.

[26] E. Braaten and A. Nieto, arXiv:hep-th/9609047, Phys`Rev. B 5, 8090 (1997).

[27] J. E. Mandula, G. Zweig and J. Govaerts, Nucl. Phys. B 228, 91 (1983).

[28] X. Li and C. Liu, Phys. Lett. B 587, 100 (2004) arXiv:hep-lat/0311035.

[29] W. Detmold and M. J. Savage, Nucl. Phys. A 743, 170 (2004) arXiv:hep-lat/0403005.

[30] X. Feng, X. Li and C. Liu, Phys. Rev. D 70, 014505 (2004) arXiv:hep-lat/0404001.

[31] E. Braaten, H. W. Hammer and S. Hermans, Phys. Rev. A 63, 063609 (2001) arXiv:cond-mat/0012043.

[32] S. R. Beane, P. F. Bedaque, A. Parreño and M. J. Savage, Phys. Lett. B 585, 106 (2004) arXiv:hep-lat/0312004.

[33] S. Sasaki and T. Yamazaki, Phys. Rev. D 74, 114507 (2006) arXiv:hep-lat/0610081. 